\title{
Spontaneous tumor lysis syndrome in a patient with metastatic prostate cancer
}

\author{
NAOMI SERLING-BOYD ${ }^{1}$, ZOE QUANDT $^{2}$ and NAZIMA ALLAUDEEN ${ }^{3}$ \\ ${ }^{1}$ Department of Medicine, Stanford Hospital and Clinics, Stanford, CA 94305; ${ }^{2}$ Department of Medicine, \\ University of California San Francisco, San Francisco, CA 94143; ${ }^{3}$ Department of Medicine, \\ VA Palo Alto Health Care System, Palo Alto, CA 94304, USA
}

Received October 6, 2016; Accepted January 10, 2017

DOI: $10.3892 /$ mco.2017.1186

\begin{abstract}
Tumor lysis syndrome (TLS) is a life-threatening oncological emergency, with most cases occurring in hematological malignancies following the initiation of treatment. However, on rare occasions, TLS may occur in solid tumors as well. In the present case study, the case is reported of a 56-year-old African-American man who presented with a recent diagnosis of prostate cancer, abdominal pain, elevated transaminases, renal insufficiency, hyperkalemia, and hyperuricemia, consistent with spontaneous TLS in the setting of metastatic prostate cancer. A computed tomography scan of the patient's abdomen demonstrated diffuse metastatic tumor burden. Following treatment with allopurinol, rasburicase, and initiation of anti-androgen therapy for the prostate cancer, the patient's TLS laboratory results normalized, however, his renal functions continued to decline. TLS is rare in solid tumors, and particularly rare in prostate cancer, with only six other case reports of the syndrome occurring to the best of our knowledge. This case report highlights the need for early recognition of TLS, even in cases that are not typically associated with the syndrome, as prompt diagnosis will affect early management and may be able to prevent or minimize complications.
\end{abstract}

\section{Introduction}

Tumor lysis syndrome (TLS) is a life-threatening complication associated with malignancies and their treatment. TLS is characterized by hyperuricemia, hyperkalemia, hyperphosphatemia, and hypocalcemia due to rapid cell death and

Correspondence to: Dr Nazima Allaudeen, Department of Medicine, VA Palo Alto Health Care System, 3801 Miranda Avenue, MC 111, Palo Alto, CA 94304, USA

E-mail: nazima.allaudeen@va.gov

Abbreviations: TLS, tumor lysis syndrome; LDH, lactate dehydrogenase; PSA, prostate specific antigen; CT, computed tomography; GnRH, gonadotrophin-releasing hormone

Key words: spontaneous tumor lysis syndrome, metastatic prostate cancer, hyperuricemia release of intracellular material. Laboratory diagnosis of TLS encompasses the four laboratory abnormalities above, and further clinical manifestations may include renal impairment, cardiac arrhythmias, seizures, and ultimately mortality. TLS most commonly occurs in hematological malignancies following the initiation of treatment, and is known to occur in solid tumors after treatment (1-3). However, it very rarely occurs spontaneously, and is even more rarely reported in cases of prostate cancer. Prompt diagnosis is critical, as management includes aggressive hydration and uric acid-lowering therapy. There is a lack of universal definition for diagnosing TLS, but the most accepted classification system is the Cairo-Bishop criteria (4). However, since this requires the abnormal laboratory values to be determined within 3 days prior to, and 7 days following, chemotherapy, it does not provide a framework for defining spontaneous TLS.

A literature search for 'tumor lysis syndrome' and 'prostate cancer' revealed six cases of TLS associated with prostate cancer (5-10). Of these six, two patients developed TLS following the initiation of cytotoxic chemotherapy, two patients after androgen blockade (both within days to weeks following treatment), one following radiation treatment, and one after have received both radiation and an androgen blockade. The mortality rate was $100 \%$ among five of the cases, and not reported in the sixth (Table I). Each of these cases was treated with aggressive supportive measures, and only one of the patients received rasburicase (10), while one received allopurinol, as per their reports (6). Rasburicase, a recombinant version of urate oxidase that metabolizes uric acid to allantoin, may cause an abrupt lowering of serum uric acid. There have been several randomized controlled trials of rasburicase use in TLS (3); however, the primary endpoint assessed in these trials was post-treatment uric acid levels, and they were not empowered to evaluate mortality or other morbidity characteristics, such as the need for dialysis.

It is unclear why some patients develop spontaneous TLS in the setting of solid tumors, but risk factors may include high levels of lactate dehydrogenase (LDH), pre-existing renal impairment, and hyperuricemia $(1,3)$. Further risk factors for TLS in solid tumors include highly proliferating and bulky malignancy, sensitivity to chemotherapy, exposure to nephrotoxic substances, and decreased fluid intake (1,3). Liver metastases are also possibly a risk factor (1). 


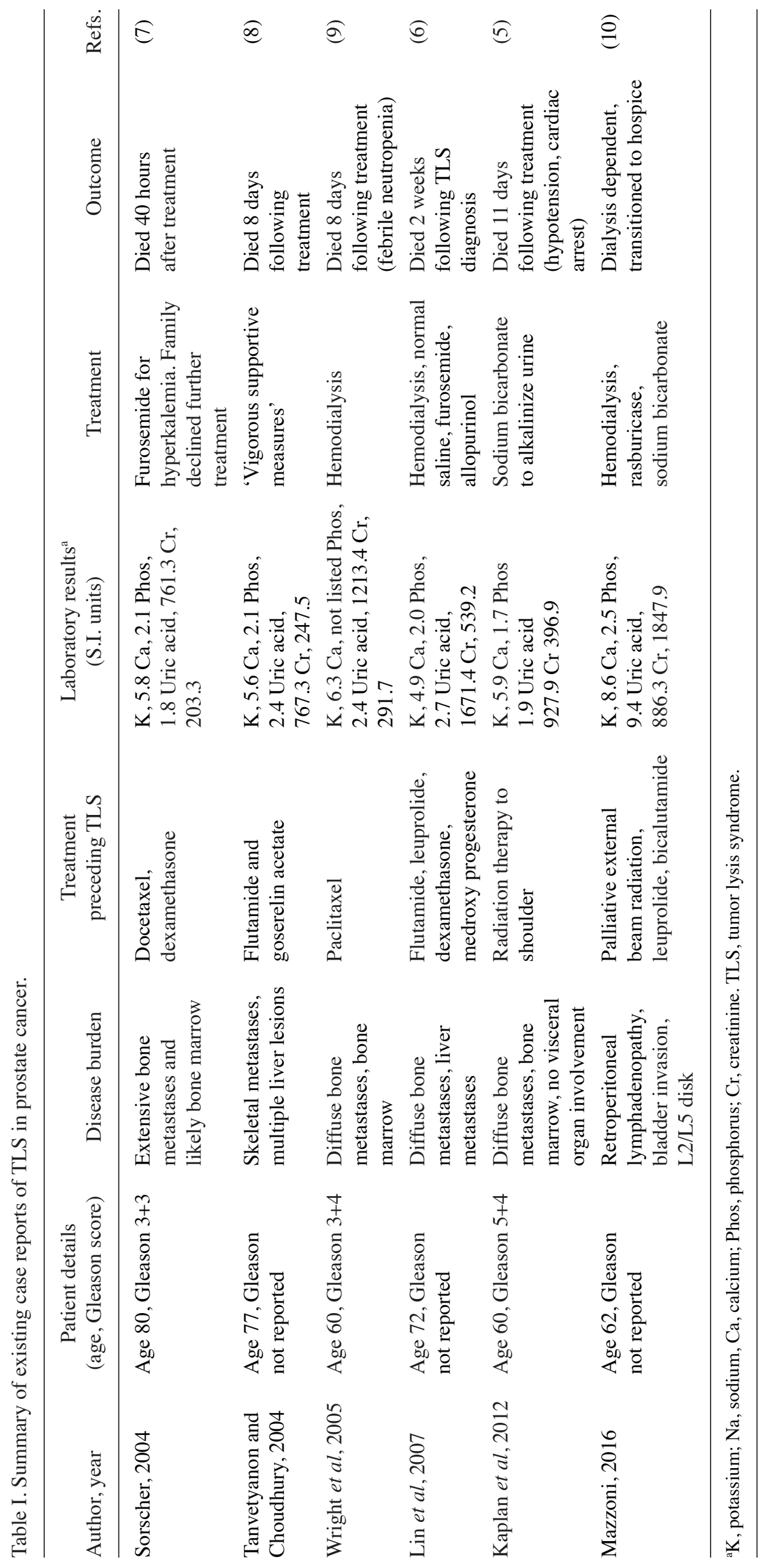


Table II. Pertinent laboratory values.

\begin{tabular}{|c|c|c|c|c|c|c|}
\hline & $\begin{array}{l}\text { Normal values } \\
\quad \text { (SI units) }\end{array}$ & Admission & $\begin{array}{l}\text { Hospital day } 2 \\
\text { (pre-rasburicase) }\end{array}$ & $\begin{array}{c}\text { Hospital day } 3 \\
\text { (post-rasburicase) }\end{array}$ & $\begin{array}{l}\text { Hospital } \\
\text { day } 4\end{array}$ & $\begin{array}{c}\text { Hospital } \\
\text { day } 12\end{array}$ \\
\hline Potassium & $3.5-5.5 \mathrm{mmol} / \mathrm{l}$ & 5.7 & 6.0 & 4.5 & 3.9 & 4.0 \\
\hline BUN & $2.1-9.3 \mathrm{mmol} / 1$ & 16.4 & 18.2 & 17.1 & 16.1 & 30.3 \\
\hline Creatinine & $35.4-114.9 \mu \mathrm{mol} / \mathrm{l}$ & 174.1 & 190.9 & 178.6 & 197.1 & 274.9 \\
\hline Calcium & $2.1-10.5 \mathrm{mmol} / 1$ & 2.5 & 2.2 & 2.2 & 2.1 & 2.2 \\
\hline Phosphorous & $2.5-2.6 \mathrm{mmol} / \mathrm{l}$ & 1.2 & 1.5 & 1.1 & 1.0 & - \\
\hline Total bilirubin & $1.7-20.5 \mu \mathrm{mol} / 1$ & 201.8 & 186.4 & 164.2 & 155.6 & 155.6 \\
\hline AST & $7-55 \mathrm{U} / 1$ & 494 & 543 & 568 & 546 & 513 \\
\hline ALT & 8-48 U/1 & 168 & 182 & 181 & 172 & 115 \\
\hline Alk. phos. & $45-115 \mathrm{U} / 1$ & 1,034 & 965 & 837 & 787 & 670 \\
\hline Uric acid & $232-535.3 \mu \mathrm{mol} / 1$ & 981.4 & 927.9 & 672.1 & 577.0 & - \\
\hline $\mathrm{LDH}$ & $60-250 \mathrm{U} / 1$ & 4,023 & 4,360 & 3,814 & 4,034 & - \\
\hline Lactate & $0.5-1.6 \mathrm{mmol} / 1$ & - & 2.1 & - & 3.8 & - \\
\hline PSA & $0-4 \mu \mathrm{mol} / 1$ & 548 & - & - & - & - \\
\hline
\end{tabular}

Where shown, the dashes indicate that the test was not performed. BUN, blood urea nitrogen; AST, aspartate transaminase; ALT, alanine transaminase; Alk. phos., alkaline phosphatase; LDH, lactate dehydrogenase; PSA, prostate specific antigen.

To the best of our knowledge, this is the first case report of spontaneous TLS in a patient with treatment naïve metastatic prostate cancer.

\section{Case report}

A 56-year-old African-American man with no known medical history presented to the emergency room of a Veterans Affairs hospital with abdominal pain and was admitted to the internal medicine service. One month previously, he had been diagnosed with prostate cancer (Gleason 5+4=9) (11) after undergoing transrectal ultrasonography as a result of obstructive urinary symptoms and a nodular firm prostate upon rectal examination at another hospital. Due to problems with insurance, the patient had not had further evaluation, and had yet to begin treatment. On presentation to our emergency department, the patient complained of epigastric abdominal pain, straining with urination, decreased appetite, weight loss, hot flashes, chills, and non-productive cough. The patient's father had prostate cancer that was diagnosed in his 70s. A physical examination was remarkable for its normal vital signs. The patient appeared tired, but was in no distress. A markedly enlarged and slightly tender liver spanning across the upper abdomen was appreciated, as well as a firm and nodular prostate.

The patient's initial laboratory results were notable for hyperkalemia, elevated creatinine levels, elevated liver function tests, and elevated prostate specific antigen (PSA) (Table II). His LDH and uric acid levels were grossly elevated. A computed tomography (CT) scan of the abdomen and pelvis revealed an enlarged prostate measuring $6.3 \times 5.1 \times 6.1 \mathrm{~cm}$, multiple lumbar spine and pelvic sclerotic lesions, multiple enlarged abdominal and pelvic lymph nodes, and marked hepatomegaly spanning $29 \mathrm{~cm}$ in the transverse dimension.

The initial laboratory tests raised a concern for TLS, although there was initial uncertainty due to lack of a typical malignancy associated with TLS. The patient was treated with aggressive intravenous fluids including normal saline boluses followed by an infusion at $300 \mathrm{cc} / \mathrm{h}$, allopurinol (300 $\mathrm{mg}$ twice daily), and then sodium bicarbonate at $200 \mathrm{cc} / \mathrm{h}$; following a discussion with the oncology department, rasburicase was initiated; he was given a single dose of $6 \mathrm{mg}$ intravenously. The patient's potassium levels normalized, the uric acid level slowly decreased to a nadir of $130.9 \mu \mathrm{mol} / 1$, and the liver function tests stabilized (his aspartate transaminase peaked at 568 and trended down to 513, and alanine transaminase peaked at 182 and trended down to 115). Positron emission tomography (PET)-CT revealed a marked metabolic activity within the prostate gland, as well as diffuse hepatic, right supraclavicular, and retroperitoneal and pelvic nodal lesions. Doppler ultrasound of the portal vein revealed a patent vessel. Due to the rare occurrence of TLS in prostate cancer, as well as the unusual presentation of dramatic hepatic infiltration, a liver biopsy was pursued to rule out possible second malignancy. Liver biopsy pathology revealed metastatic adenocarcinoma consistent with prostate gland origin, based on immunohistochemical staining patterns. On hospital day 2, the patient began a course of treatment with degarelix, a gonadotrophin-releasing hormone (GnRH) antagonist. He received $240 \mathrm{mg}$ subcutaneously, and received a second dose of $80 \mathrm{mg}$ one month later. The patient's tumor lysis laboratory results continued to normalize. However, the patient's renal and liver failure slowly progressed, along with waxing and waning mental status attributed to encephalopathy. The patient had ongoing abdominal pain, nausea, and further functional deterioration. After multiple evolving goals of care discussions with the patient and his family, he was transitioned to inpatient hospice care on hospital day 20 , and succumbed to mortality 6 weeks afterwards. Informed consent was obtained from the patient for the publication of this case report. 


\section{Discussion}

TLS seldom occurs outside of the setting of treating hematological malignancies, and rarely occurs in solid tumors (1-3). Even more rare is the spontaneous development of TLS (2), and, to the best of our knowledge, this is the first case report of spontaneous TLS in prostate cancer in a treatment-naïve patient. Although the Cairo-Bishop criteria (4) are not intended to diagnose spontaneous TLS, our patient met the criteria for a laboratory diagnosis of TLS with hyperphosphatemia, hyperuricemia, and hyperkalemia. The patient additionally had renal impairment, with a creatinine level that rose to two times above the baseline, placing him in the Grade II classification of the Cairo-Bishop criteria (4). The patient did not suffer from any cardiac arrhythmias or seizures.

As described, this is the first case of spontaneous TLS in a treatment-naïve patient (Table I). Our patient had a large number of the risk factors for TLS, including bulky disease, high LDH levels, pre-existing renal disease and liver metastases. Additionally, while each of the prior cases was treated with aggressive supportive measures, only one of the patients received rasburicase (10) and one received allopurinol (6). Although our patient's laboratory values are in line with those of the other patients, he surpassed the survival of most of the other patients in the reported cases, perhaps in part due to the rapid recognition and aggressive treatment of the patient's TLS and the use of rasburicase, although this has yet to be studied in association with mortality.

Prompt recognition of any laboratory abnormalities and consideration of TLS are essential for any patient presenting with significant tumor burden, either prior to or following treatment. One may even consider checking the baseline uric acid and LDH levels as part of an initial workup of any new malignancy, regardless of the type. Further studies are necessary to identify particular clinical characteristics or laboratory values associated with significantly higher risk of cancer. Prophylaxis with allopurinol and frequent laboratory monitoring may be warranted in these patients.

\section{Acknowledgements}

We would like to thank our patient for agreeing to be a part of this clinical vignette. Views expressed are those of the authors and not necessarily those of the Department of Veterans Affairs.

\section{References}

1. Mirrakhimov AE, Ali AM, Khan M and Barbaryan A: Tumor lysis syndrome in solid tumors: An up to date review of the literature. Rare Tumors 6: 5389, 2014.

2. Vaisban E, Braester A, Mosenzon O, Kolin M and Horn Y: Spontaneous tumor lysis in solid tumors: Really a rare condition? Am J Med Sci 325: 38-40, 2003.

3. Wilson FP and Berns JS: Onco-Nephrology: Tumor lysis syndrome. Clin J Am Soc Nephrol 7: 1730-1739, 2012.

4. Cairo MS and Bishop M: Tumor lysis syndrome: New therapeutic strategies and classification. Br J Haematol 127: 3-11, 2004.

5. Kaplan MA, Kucukoner M, Alpagat G and Isikdogan A: Tumor lysis syndrome during radiotherapy for prostate cancer with bone and bone marrow metastases without visceral metastasis. Ann Saudi Med 32: 306-308, 2012.

6. Lin CJ, Hsieh RK, Lim KH, Chen HH, Cheng YC and Wu CJ: Fatal spontaneous tumor lysis syndrome in a patient with metastatic, androgen-independent prostate cancer. South Med J 100: 916-917, 2007.

7. Sorscher SM: Tumor lysis syndrome following docetaxel therapy for extensive metastatic prostate cancer. Cancer Chemother Pharmacol 54: 191-192, 2004.

8. Tanvetyanon T and Choudhury AM: Fatal acute tumor lysis syndrome, hepatic encephalopathy and flare phenomenon following combined androgen blockade. J Urol 171: 1627, 2004.

9. Wright JL, Lin DW, Dewan P and Montgomery RB: Tumor lysis syndrome in a patient with metastatic, androgen independent prostate cancer. Int J Urol 12: 1012-1013, 2005.

10. Mazzoni S: Tumor lysis syndrome in anti-androgen-treated metastatic prostate cancer. Int Urol Nephrol 48: 1837-1838, 2016.

11. Epstein JI, Egevad L, Amin MB, Delahunt B, Srigley JR and Humphrey PA; Grading Committee: The 2014 International Society of Urological Pathology (ISUP) Consensus Conference on Gleason Grading of Prostatic Carcinoma: Definition of Grading Patterns and Proposal for a New Grading System. Am J Surg Pathol 40: 244-252, 2016. 